\title{
Copper-catalyzed trifluoromethylation of alkenes with an electrophilic trifluoromethylating reagent
}

\author{
Xiao-Ping Wang ${ }^{1,2}$, Jin-Hong Lin ${ }^{1}$, Cheng-Pan Zhang ${ }^{1}$, Ji-Chang Xiao ${ }^{* 1}$ \\ and Xing Zheng ${ }^{2}$
}

Open Access

\author{
Letter \\ Address: \\ ${ }^{1}$ Key Laboratory of Organofluorine Chemistry, Shanghai Institute of \\ Organic Chemistry, Chinese Academy of Sciences,345 Lingling Road, \\ Shanghai 200032, China and ${ }^{2}$ Institute of Pharmacy and \\ Pharmacology, University of South China, Hengyang, Hunan 421001, \\ China \\ Email: \\ Ji-Chang Xiao* - jchxiao@sioc.ac.cn \\ * Corresponding author \\ Keywords: \\ alkenes; catalysis; copper; electrophilic trifluoromethylating reagent; \\ trifluoromethylation
}

Beilstein J. Org. Chem. 2013, 9, 2635-2640. doi:10.3762/bjoc. 9.299

Received: 18 July 2013

Accepted: 06 November 2013

Published: 25 November 2013

This article is part of the Thematic Series "Organo-fluorine chemistry III".

Guest Editor: D. O'Hagan

(c) 2013 Wang et al; licensee Beilstein-Institut. License and terms: see end of document.

\begin{abstract}
An efficient method for the copper-catalyzed trifluoromethylation of terminal alkenes with an electrophilic trifluoromethylating reagent has been developed. The reactions proceeded smoothly to give trifluoromethylated alkenes in good to excellent yields. The results provided a versatile approach for the construction of $\mathrm{C}_{\mathrm{vinyl}}-\mathrm{CF}_{3}$ bonds without using prefunctionalized substrates.
\end{abstract}

\section{Introduction}

The incorporation of a trifluoromethyl group into pharmaceutically and agrochemically relevant molecules usually enhances their chemical and metabolic stability, lipophilicity and binding selectivity [1-7]. As a result, considerable effort has been directed towards the development of efficient and versatile trifluoromethylation methods [8-16]. The past few years has witnessed the rapid advances in transition metal-promoted trifluoromethylation for the construction of $\mathrm{C}_{\text {aryl }}-\mathrm{CF}_{3}$ bonds [1731]. In contrast, transition metal-mediated trifluoromethylation to form $\mathrm{C}_{\mathrm{vinyl}}-\mathrm{CF}_{3}$ bonds has been much less explored. As illustrated in Scheme 1, the strategies developed recently usually require the use of prefunctionalized alkenes, which could be classified into the following: vinylboronic acids, vinyl borates, vinyl halides, vinyl sulfonates and vinyl carboxylic acids (Scheme 1, reaction 1) [32-38]. Cho and co-workers reported a radical process for the trifluoromethylation of terminal alkenes without using prefunctionalized substrates, but a volatile reagent was used (Scheme 1, reaction 1) [39]. Szabó described trifluoromethyl-benzoyloxylation of alkynes to construct $\mathrm{C}_{\text {vinyl }}-\mathrm{CF}_{3}$ bonds (Scheme 2, reaction 2) [40]. As part of our continuing interest in trifluoromethylation reactions [25,41-45], we investigated the copper-catalyzed trifluoromethylation of terminal alkenes with electrophilic trifluoromethylating reagents (Scheme 1, reaction 3). 


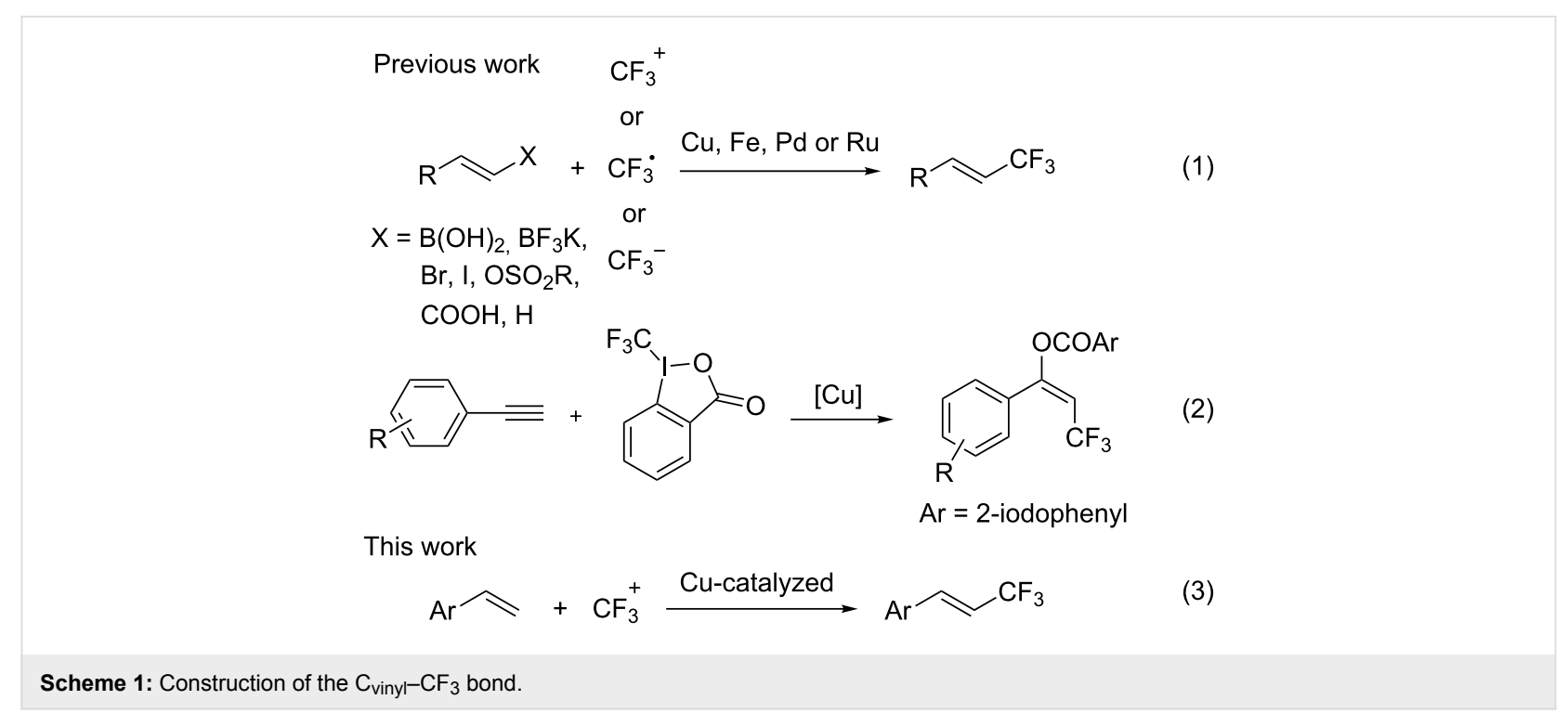

Transition metal-catalyzed trifluoromethylation of terminal alkenes has been reported by several groups. However, these methods focused on the formation of $\mathrm{C}_{\mathrm{sp} 3}-\mathrm{CF}_{3}$ bonds [46-56] In copper-catalyzed reactions with electrophilic trifluoromethylating reagents, it was proposed that the transformation might proceed via a radical, electrophilic or Heck-type process (Scheme 2) [47-50]. We reasoned that even if the reaction involved the radical process (path A, Scheme 2), the radical intermediate could still be oxidized to a cation because electrophilic trifluoromethylation reagents can be considered as an oxidant. In the presence of base, both of the cation and Hecktype intermediates should be able to undergo hydrogen elimination to form a $\mathrm{C}_{\mathrm{vinyl}}-\mathrm{CF}_{3}$ bond. On the basis of these reports and our hypothesis, we commenced to examine the reaction of aromatic alkenes with electrophilic trifluoromethylation reagents in the presence of copper and base.

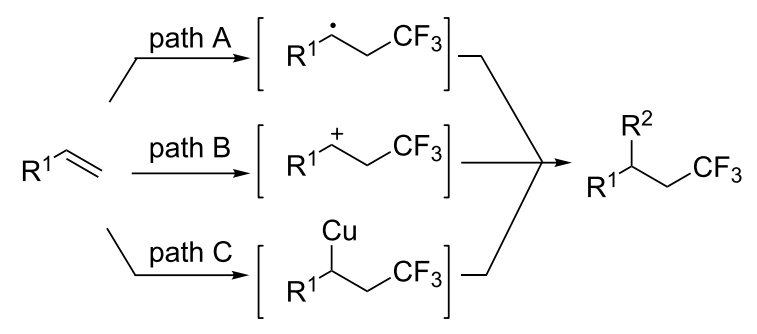

Scheme 2: Proposed reaction paths for the trifluoromethylation of alkenes.

\section{Results and Discussion}

Previously, we reported that copper powder or cuprous iodide could promote trifluoromethylation of heteroaromatics, arylboronic acids or terminal alkynes with trifluoromethyl sulfo- nium salt I $[25,44,45,57,58]$. But the same trifluoromethylation reagent ( 2 equiv) failed to convert 4 -vinylbiphenyl to the expected alkene in reasonable yields in acetonitrile with $\mathrm{B}_{1}$ (DBU) as the base, even though cuprous iodide gave better results (Table 1, entries 1 and 2). In the presence of cuprous iodide, Umemoto's reagent (II) and Togni's reagent (III) were found to be more efficient in this transformation (Table 1, entries 3 and 4). ${ }^{19} \mathrm{~F}$ NMR measurements showed that the reaction system turned to be complex and low yield of the desired product was determined when another Togni's reagent (IV) was used (Table 1, entry 5). Other cuprous complexes were also studied with the use of Togni's reagent (III) (Table 1, entries $6-10)$. Better result obtained with $\left[(\mathrm{MeCN})_{4} \mathrm{Cu}\right] \mathrm{PF}_{6}$ prompted us to continue to use this copper source (Table 1, entry 10). When $\mathrm{B}_{2}$ was used instead of $\mathrm{B}_{1}$, almost no desired product was observed (Table 1 , entry 11 ). That might be because $\mathrm{B}_{2}$ not only acted as base, but also acted as strongly coordinating ligand, which poisoned the catalyst and shut down the reaction. Other bases (Table 1, entries 12-15), including an inorganic base (Table 1, entry 15), failed to accelerate the desired conversion either. The examination of the solvent effect showed that the solvent is quite important for the reaction (Table 1, entries 16-20). When the reaction was carried out in DMF, the expected product 2a was obtained in excellent yield (Table 1, entry 16). The yield was decreased dramatically in DMSO (Table 1, entry 17). Almost no reaction took place in polar protic solvent (Table 1, entry 18) and moderate results were achieved in less polar solvents (Table 1, entries 19 and 20). With increasing the amount of Togni's reagent (III) in the suitable solvent, DMF, the product was obtained almost in quantitative yield with excellent stereoselectivity ( $\mathrm{dr}>98: 2)$ determined by ${ }^{19}$ F NMR (Table 1, entry 21 ). Decreasing the amount of this reagent resulted in a lower yield (Table 1, entry 22). The 
Table 1: Trifluoromethylation of 4-vinylbiphenyl by electrophilic trifluoromethylation reagents.

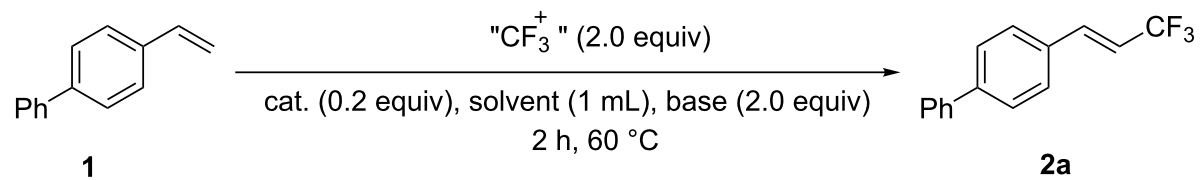

1 $2 \mathrm{~h}, 60^{\circ} \mathrm{C}$

$2 a$

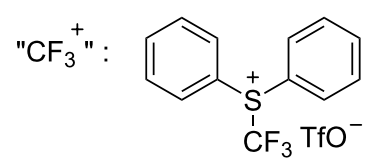

I<smiles>[O-][c-]1c2ccccc2c2ccccc21</smiles>

II<smiles>CC1(C)OI(c2ccccc2C(F)(F)F)C1(C)C</smiles>

III<smiles>O=C1OI(C(F)(F)F)c2ccccc21</smiles>

IV

base:<smiles>C1CCC2=NCCCN2CC1</smiles><smiles>CCN(CC)CC</smiles><smiles>CC(C)NC(C)C</smiles>

$\mathrm{NaOAc}$

$B_{1}$

$\mathrm{B}_{2}$

$\mathrm{B}_{3}$

$\mathrm{B}_{4}$

$\mathrm{B}_{5} \quad \mathrm{~B}_{6}$

\begin{tabular}{|c|c|c|c|c|c|}
\hline Entry & Cat. & Base & Solvent & $" \mathrm{CF}_{3}{ }^{+} "$ & Yield $(\%)^{a}$ \\
\hline 1 & $\mathrm{Cu}$ & $\mathrm{B}_{1}$ & $\mathrm{CH}_{3} \mathrm{CN}$ & I & trace \\
\hline 2 & Cul & $\mathrm{B}_{1}$ & $\mathrm{CH}_{3} \mathrm{CN}$ & I & 30 \\
\hline 3 & Cul & $\mathrm{B}_{1}$ & $\mathrm{CH}_{3} \mathrm{CN}$ & II & 56 \\
\hline 4 & Cul & $\mathrm{B}_{1}$ & $\mathrm{CH}_{3} \mathrm{CN}$ & III & 60 \\
\hline 5 & Cul & $\mathrm{B}_{1}$ & $\mathrm{CH}_{3} \mathrm{CN}$ & IV & 30 \\
\hline 6 & $\mathrm{CuBr}$ & $\mathrm{B}_{1}$ & $\mathrm{CH}_{3} \mathrm{CN}$ & III & 46 \\
\hline 7 & $\mathrm{CuCl}$ & $\mathrm{B}_{1}$ & $\mathrm{CH}_{3} \mathrm{CN}$ & III & 36 \\
\hline 8 & CuTc & $\mathrm{B}_{1}$ & $\mathrm{CH}_{3} \mathrm{CN}$ & III & 54 \\
\hline 9 & CuOAc & $\mathrm{B}_{1}$ & $\mathrm{CH}_{3} \mathrm{CN}$ & III & 41 \\
\hline 10 & {$\left[(\mathrm{MeCN})_{4} \mathrm{Cu}\right] \mathrm{PF}_{6}$} & $\mathrm{~B}_{1}$ & $\mathrm{CH}_{3} \mathrm{CN}$ & III & 75 \\
\hline 11 & {$\left[(\mathrm{MeCN})_{4} \mathrm{Cu}\right] \mathrm{PF}_{6}$} & $\mathrm{~B}_{2}$ & $\mathrm{CH}_{3} \mathrm{CN}$ & III & trace \\
\hline 12 & {$\left[(\mathrm{MeCN})_{4} \mathrm{Cu}\right] \mathrm{PF}_{6}$} & $\mathrm{~B}_{3}$ & $\mathrm{CH}_{3} \mathrm{CN}$ & III & 10 \\
\hline 13 & {$\left[(\mathrm{MeCN})_{4} \mathrm{Cu}\right] \mathrm{PF}_{6}$} & $\mathrm{~B}_{4}$ & $\mathrm{CH}_{3} \mathrm{CN}$ & III & trace \\
\hline 14 & {$\left[(\mathrm{MeCN})_{4} \mathrm{Cu}\right] \mathrm{PF}_{6}$} & $\mathrm{~B}_{5}$ & $\mathrm{CH}_{3} \mathrm{CN}$ & III & 10 \\
\hline 15 & {$\left[(\mathrm{MeCN})_{4} \mathrm{Cu}\right] \mathrm{PF}_{6}$} & $\mathrm{~B}_{6}$ & $\mathrm{CH}_{3} \mathrm{CN}$ & III & 7 \\
\hline 16 & {$\left[(\mathrm{MeCN})_{4} \mathrm{Cu}\right] \mathrm{PF}_{6}$} & $\mathrm{~B}_{1}$ & DMF & III & 90 \\
\hline 17 & {$\left[(\mathrm{MeCN})_{4} \mathrm{Cu}\right] \mathrm{PF}_{6}$} & $\mathrm{~B}_{1}$ & DMSO & III & 57 \\
\hline 18 & {$\left[(\mathrm{MeCN})_{4} \mathrm{Cu}\right] \mathrm{PF}_{6}$} & $\mathrm{~B}_{1}$ & $\mathrm{CH}_{3} \mathrm{OH}$ & III & trace \\
\hline 19 & {$\left[(\mathrm{MeCN})_{4} \mathrm{Cu}\right] \mathrm{PF}_{6}$} & $\mathrm{~B}_{1}$ & THF & III & 67 \\
\hline 20 & {$\left[(\mathrm{MeCN})_{4} \mathrm{Cu}\right] \mathrm{PF}_{6}$} & $\mathrm{~B}_{1}$ & $\mathrm{CHCl}_{3}$ & III & 75 \\
\hline $21^{b}$ & {$\left[(\mathrm{MeCN})_{4} \mathrm{Cu}\right] \mathrm{PF}_{6}$} & $\mathrm{~B}_{1}$ & DMF & III & 98 \\
\hline $22^{c}$ & {$\left[(\mathrm{MeCN})_{4} \mathrm{Cu}\right] \mathrm{PF}_{6}$} & $\mathrm{~B}_{1}$ & DMF & III & 58 \\
\hline 23 & - & - & DMF & III & 0 \\
\hline 24 & - & $\mathrm{B}_{1}$ & DMF & III & 0 \\
\hline 25 & {$\left[(\mathrm{MeCN})_{4} \mathrm{Cu}\right] \mathrm{PF}_{6}$} & - & DMF & III & 10 \\
\hline
\end{tabular}

aYields determined by ${ }^{19} \mathrm{~F}$ NMR spectroscopy. ${ }^{\mathrm{b}} 2.5$ equiv of III was used. ${ }^{\mathrm{c}} 1.5$ equiv of III was used.

absence of catalyst or base led to no reaction or incredibly low yield, which means both of them are crucial for the reaction (Table 1, entries 23-25).

With the optimized reaction conditions in hand (Table 1 , entry $21)$, we then explored the substrate scope of the $\mathrm{Cu}(\mathrm{I})$-catalyzed trifluoromethylation of terminal alkenes with Togni's reagent. As shown in Figure 1, the reaction could tolerate various functional groups. It is worth mentioning that all of the products were obtained with excellent stereoselectivity $(E / Z>97 / 3)$, determined by ${ }^{19}$ F NMR. Substrates with an electron-donating group were converted smoothly into the desired products in 


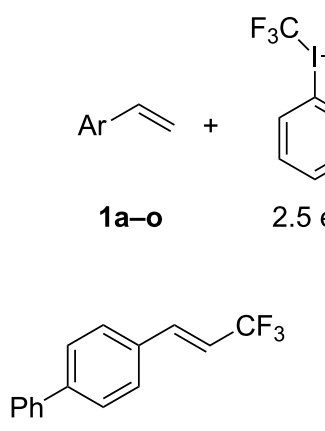

2a, $90 \%$<smiles>Cc1ccc(C=CC(F)(F)F)cc1</smiles>

$2 e, 92 \%$<smiles>FC(F)(F)/C=C/c1ccc(Br)cc1</smiles>

2i, $93 \%$<smiles>COc1ccc(/C=C/C(F)(F)F)cc1</smiles>

2b, $91 \%$

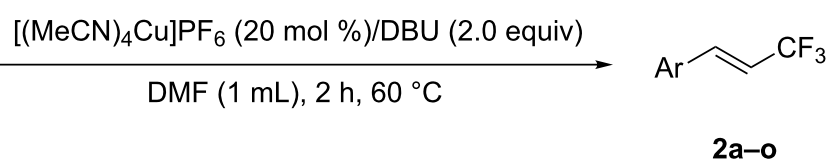<smiles>COc1ccc2cc(/C=C/C(F)(F)F)ccc2c1</smiles>

2c, $88 \%$

2d, $88 \%$<smiles>FC(F)(F)C=Cc1ccc2ccccc2c1</smiles>

2f, $92 \%$<smiles>FC(F)(F)/C=C/c1ccccc1Br</smiles>

2g, $93 \%$<smiles>FC(F)(F)/C=C/c1cccc(Br)c1</smiles>

2h, $92 \%$<smiles>FC(F)(F)C=Cc1ccc(Cl)cc1</smiles>

2j, $86 \%$<smiles>Fc1cccc(/C=C/C(F)(F)F)c1</smiles>

2k, $63 \%$<smiles>O=Cc1cccc(/C=C/C(F)(F)F)c1</smiles>

2I, $84 \%$<smiles>O=[N+]([O-])c1ccc(/C=C/C(F)(F)F)cc1</smiles>

$2 \mathrm{~m}, 76 \%$<smiles>FC(F)(F)/C=C/c1csc2ccccc12</smiles>

2n, $89 \%$<smiles>FC(F)(F)/C=C/c1cccc(Br)n1</smiles>

2o, $70 \%$

Figure 1: $\mathrm{Cu}(\mathrm{I})$-catalyzed trifluoromethylation of terminal alkenes with Togni's reagent. Isolated yield are recorded.

excellent yields $(\mathbf{2 a}-\mathbf{2} \mathbf{f})$. Irrespective of the position of the bromine substituent on the aryl ring, the reaction proceeded very well to afford the desired products in excellent yields (2g-2i). Stronger electron-withdrawing groups showed some negative effect on the reaction, as exemplified by the poor results of substrates with other halides, carbonyl or nitro groups on the benzene ring $(\mathbf{2} \mathbf{j}-\mathbf{2 m})$. Heteroaromatic alkenes were also investigated (2n and 2o). As previously seen, alkene 1n endowed with an electron-rich heteroaromatic group led to a good result (2n) and an electron-deficient substrate resulted in low yield (2o).

With regard to the reaction mechanism, it is reasonable to conceive a pathway involving radical species according to previous reports [47-50]. To gain more insight into the mechanism, further evidence was collected. 2,2,6,6-Tetramethyl-1piperidinyloxy (TEMPO), a well-known radical scavenger, was added to the reaction of 4-vinylbiphenyl with Togni's reagent (III) in the presence of $\left[(\mathrm{MeCN})_{4} \mathrm{Cu}_{3} \mathrm{PF}_{6}\right.$. It was found that the desired trifluoromethylation was completely suppressed, which suggested that the transformation involved a radical process. Based on the above results, we proposed the mechanism as outlined in Scheme 3. Initially, the activation of III by $\mathrm{Cu}(\mathrm{I})$ led to the formation of radical intermediate A. Decomposition of this intermediate produces ((2-(2-iodophenyl)propan-2yl)oxy)copper(II) (C) and a $\mathrm{CF}_{3}$ radical, which is trapped by alkenes to form the trifluoromethylated radical intermediate $\mathbf{B}$. Subsequently, the radical intermediate $\mathbf{B}$ is oxidized by $\mathrm{Cu}(\mathrm{II})$ (C) to the cation intermediate $\mathbf{D}$ with simultaneous release of catalyst $\mathrm{Cu}(\mathrm{I})$. In the presence of base, intermediate $\mathbf{D}$ readily undergoes hydrogen elimination to give the final product.

\section{Conclusion}

In conclusion, we have described the copper-catalyzed trifluoromethylation of alkenes with Togni's reagent under mild conditions. The results presented here provided a versatile approach for the construction of $\mathrm{C}_{\mathrm{vinyl}}-\mathrm{CF}_{3}$ bonds without using prefunctionalized substrates. Investigations on the application of the trifluoromethylation method to the synthesis of pharmaceuticals and agrochemicals are currently underway. 


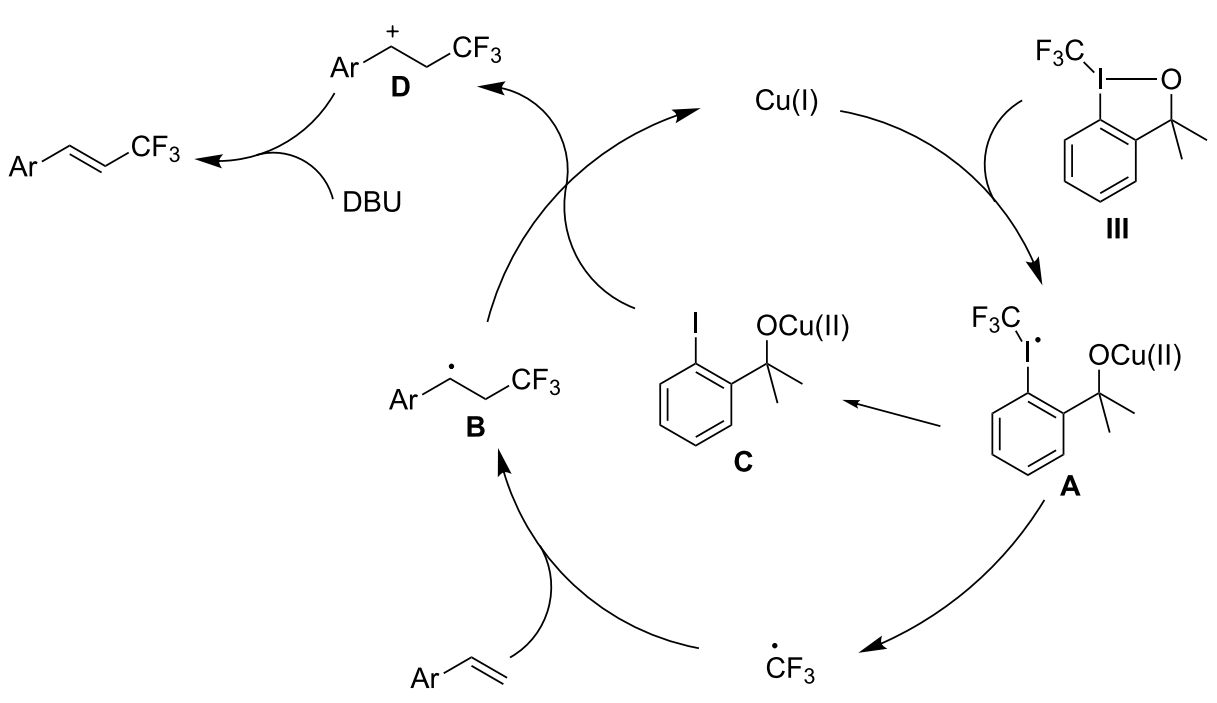

Scheme 3: Proposed mechanism for the trifluoromethylation of terminal alkenes.

\section{Supporting Information}

\section{Supporting Information File 1}

Full experimental details, analytical data and spectra of the target compounds.

[http://www.beilstein-journals.org/bjoc/content/ supplementary/1860-5397-9-299-S1.pdf]

\section{Acknowledgements}

We thank the National Natural Science Foundation of China (21032006, 21172240), the 973 Program of China (No. 2012CBA01200), the Chinese Academy of Sciences for financial support.

\section{References}

1. Kirsch, P. Modern Fluoroorganic Chemistry Synthesis, Reactivity, Applications; Wiley-VCH: Weinheim, 2004. doi:10.1002/352760393X

2. Bégué, J.-P.; Bonnet-Delpon, D. Bioorganic and Medicinal Chemistry of Fluorine; John Wiley \& Sons: Hoboken, 2008. doi:10.1002/9780470281895

3. Schlosser, M. Angew. Chem., Int. Ed. 2006, 45, 5432-5446. doi:10.1002/anie.200600449

4. Müller, K.; Faeh, C.; Diederich, F. Science 2007, 317, 1881-1886. doi:10.1126/science. 1131943

5. Hagmann, W. K. J. Med. Chem. 2008, 51, 4359-4369. doi:10.1021/jm800219f

6. Purser, S.; Moore, P. R.; Swallow, S.; Gouverneur, V. Chem. Soc. Rev. 2008, 37, 320-330. doi:10.1039/b610213c

7. Furuya, T.; Kamlet, A. S.; Ritter, T. Nature 2011, 473, 470-477. doi:10.1038/nature10108

8. Zheng, Y.; Ma, J.-A. Adv. Synth. Catal. 2010, 352, 2745-2750. doi:10.1002/adsc.201000545
9. Nie, J.; Guo, H.-C.; Cahard, D.; Ma, J.-A. Chem. Rev. 2010, 111, 455-529. doi:10.1021/cr100166a

10. Parsons, A. T.; Buchwald, S. L. Nature 2011, 480, 184-185. doi:10.1038/480184a

11. Studer, A. Angew. Chem., Int. Ed. 2012, 51, 8950-8958. doi:10.1002/anie.201202624

12. Nagib, D. A.; MacMillan, D. W. C. Nature 2011, 480, 224-228. doi:10.1038/nature10647

13. Pham, P. V.; Nagib, D. A.; MacMillan, D. W. C. Angew. Chem., Int. Ed. 2011, 50, 6119-6122. doi:10.1002/anie.201101861

14. Prakash, G. K. S.; Jog, P. V.; Batamack, P. T. D.; Olah, G. A. Science 2012, 338, 1324-1327. doi:10.1126/science.1227859

15. Pair, E.; Monteiro, N.; Bouyssi, D.; Baudoin, O. Angew. Chem., Int. Ed. 2013, 52, 5346-5349. doi:10.1002/anie.201300782

16. Wang, X.; Xu, Y.; Mo, F.; Ji, G.; Quu, D.; Feng, J.; Ye, Y.; Zhang, S.; Zhang, Y.; Wang, J. J. Am. Chem. Soc. 2013, 135, 10330-10333. doi:10.1021/ja4056239

17. Lundgren, R. J.; Stradiotto, M. Angew. Chem., Int. Ed. 2010, 49, 9322-9324. doi:10.1002/anie.201004051

18. Tomashenko, O. A.; Grushin, V. V. Chem. Rev. 2011, 111, 4475-4521. doi:10.1021/cr1004293

19. Besset, T.; Schneider, C.; Cahard, D. Angew. Chem., Int. Ed. 2012, 51, 5048-5050. doi:10.1002/anie.201201012

20. Wu, X.-F.; Neumann, H.; Beller, M. Chem.-Asian J. 2012, 7, 1744-1754. doi:10.1002/asia.201200211

21. Oishi, M.; Kondo, H.; Amii, H. Chem. Commun. 2009, 1909-1911. doi:10.1039/b823249k

22. Cho, E. J.; Senecal, T. D.; Kinzel, T.; Zhang, Y.; Watson, D. A.; Buchwald, S. L. Science 2010, 328, 1679-1681. doi:10.1126/science.1190524

23. Wang, X.; Truesdale, L.; Yu, J.-Q. J. Am. Chem. Soc. 2010, 132, 3648-3649. doi:10.1021/ja909522s

24. Chu, L.; Qing, F.-L. J. Am. Chem. Soc. 2012, 134, 1298-1304. doi:10.1021/ja209992w

25. Zhang, C.-P.; Wang, Z.-L.; Chen, Q.-Y.; Zhang, C.-T.; Gu, Y.-C.; Xiao, J.-C. Angew. Chem., Int. Ed. 2011, 50, 1896-1900. doi:10.1002/anie.201006823 
26. Zhang, X.-G.; Dai, H.-X.; Wasa, M.; Yu, J.-Q. J. Am. Chem. Soc. 2012, 134, 11948-11951. doi:10.1021/ja305259n

27. Herrmann, A. T.; Smith, L. L.; Zakarian, A. J. Am. Chem. Soc. 2012, 134, 6976-6979. doi:10.1021/ja302552e

28. Liu, T.; Shao, X.; Wu, Y.; Shen, Q. Angew. Chem., Int. Ed. 2012, 51, 540-543. doi:10.1002/anie.201106673

29. Ye, Y.; Sanford, M. S. J. Am. Chem. Soc. 2012, 134, 9034-9037. doi:10.1021/ja301553c

30. Zeng, Y.; Zhang, L.; Zhao, Y.; Ni, C.; Zhao, J.; Hu, J. J. Am. Chem. Soc. 2013, 135, 2955-2958. doi:10.1021/ja312711c

31.Dai, J.-J.; Fang, C.; Xiao, B.; Yi, J.; Xu, J.; Liu, Z.-J.; Lu, X.; Liu, L.; Fu, Y. J. Am. Chem. Soc. 2013, 135, 8436-8439. doi:10.1021/ja404217t

32. Cho, E. J.; Buchwald, S. L. Org. Lett. 2011, 13, 6552-6555. doi:10.1021/ol202885w

33. Hafner, A.; Bräse, S. Adv. Synth. Catal. 2011, 353, 3044-3048. doi:10.1002/adsc.201100528

34. Parsons, A. T.; Senecal, T. D.; Buchwald, S. L. Angew. Chem., Int. Ed. 2012, 51, 2947-2950. doi:10.1002/anie.201108267

35. He, Z.; Luo, T.; Hu, M.; Cao, Y.; Hu, J. Angew. Chem., Int. Ed. 2012, 51, 3944-3947. doi:10.1002/anie.201200140

36. Yasu, Y.; Koike, T.; Akita, M. Chem. Commun. 2013, 49, 2037-2039. doi:10.1039/c3cc39235j

37. Li, Z.; Cui, Z.; Liu, Z.-Q. Org. Lett. 2013, 15, 406-409. doi:10.1021/ol3034059

38. Li, Y.; Wu, L.; Neumann, H.; Beller, M. Chem. Commun. 2013, 49, 2628-2630. doi:10.1039/c2cc36554e

39. Iqbal, N.; Choi, S.; Kim, E.; Cho, E. J. J. Org. Chem. 2012, 77, 11383-11387. doi:10.1021/jo3022346

40. Janson, P. G.; Ghoneim, I.; Ilchenko, N. O.; Szabó, K. J. Org. Lett. 2012, 14, 2882-2885. doi:10.1021/ol3011419

41. Lin, J.-H.; Xiao, J.-C. Eur. J. Org. Chem. 2011, 4536-4539. doi:10.1002/ejoc.201100683

42. Lin, J.-H.; Zong, G.; Du, R.-B.; Xiao, J.-C.; Liu, S. Chem. Commun. 2012, 48, 7738-7740. doi:10.1039/c2cc18064b

43. Zhang, C.-P.; Wang, Z.-L.; Chen, Q.-Y.; Zhang, C.-T.; Gu, Y.-C.; Xiao, J.-C. Chem. Commun. 2011, 47, 6632-6634. doi:10.1039/c1cc11765c

44.Zhang, C.-P.; Cai, J.; Zhou, C.-B.; Wang, X.-P.; Zheng, X.; Gu, Y.-C.; Xiao, J.-C. Chem. Commun. 2011, 47, 9516-9518. doi:10.1039/c1cc13460d

45. Wang, X.; Lin, J.; Zhang, C.; Xiao, J.; Zheng, X. Chin. J. Chem. 2013, 31, 915-920. doi:10.1002/cjoc.201300393

46. Mu, X.; Wu, T.; Wang, H.-Y.; Guo, Y.-L.; Liu, G. J. Am. Chem. Soc. 2012, 134, 878-881. doi:10.1021/ja210614y

47. Parsons, A. T.; Buchwald, S. L. Angew. Chem., Int. Ed. 2011, 50, 9120-9123. doi:10.1002/anie.201104053

48. Wang, X.; Ye, Y.; Zhang, S.; Feng, J.; Xu, Y.; Zhang, Y.; Wang, J. J. Am. Chem. Soc. 2011, 133, 16410-16413. doi:10.1021/ja207775a

49. Xu, J.; Fu, Y.; Luo, D.-F.; Jiang, Y.-Y.; Xiao, B.; Liu, Z.-J.; Gong, T.-J.; Liu, L. J. Am. Chem. Soc. 2011, 133, 15300-15303. doi:10.1021/ja206330m

50. Zhu, R.; Buchwald, S. L. J. Am. Chem. Soc. 2012, 134, 12462-12465. doi:10.1021/ja305840g

51. Miyake, Y.; Ota, S.-I.; Nishibayashi, Y. Chem.-Eur. J. 2012, 18, 13255-13258. doi:10.1002/chem.201202853

52. Yasu, Y.; Koike, T.; Akita, M. Angew. Chem., Int. Ed. 2012, 51, 9567-9571. doi:10.1002/anie.201205071
53. Egami, H.; Shimizu, R.; Kawamura, S.; Sodeoka, M Angew. Chem., Int. Ed. 2013, 52, 4000-4003. doi:10.1002/anie.201210250

54. Liu, X.; Xiong, F.; Huang, X.; Xu, L.; Li, P.; Wu, X. Angew. Chem., Int. Ed. 2013, 52, 6962-6966. doi:10.1002/anie.201302673

55. Wu, X.; Chu, L.; Qing, F.-L. Angew. Chem., Int. Ed. 2013, 52, 2198-2202. doi:10.1002/anie.201208971

56. Mizuta, S.; Verhoog, S.; Engle, K. M.; Khotavivattana, T.; O’Duill, M.; Wheelhouse, K.; Rassias, G.; Médebielle, M.; Gouverneur, V. J. Am. Chem. Soc. 2013, 135, 2505-2508. doi:10.1021/ja401022x

57. Yang, J.-J.; Kirchmeier, R. L.; Shreeve, J. M. J. Org. Chem. 1998, 63, 2656-2660. doi:10.1021/jo972213।

See for some references on $S$-tifluoromethyl sulfonium salt.

58. Magnier, E.; Blazejewski, J.-C.; Tordeux, M.; Wakselman, C. Angew. Chem., Int. Ed. 2006, 45, 1279-1282. doi:10.1002/anie.200503776

See for some references on S-tifluoromethyl sulfonium salt.

\section{License and Terms}

This is an Open Access article under the terms of the Creative Commons Attribution License (http://creativecommons.org/licenses/by/2.0), which permits unrestricted use, distribution, and reproduction in any medium, provided the original work is properly cited.

The license is subject to the Beilstein Journal of Organic Chemistry terms and conditions:

(http://www.beilstein-journals.org/bjoc)

The definitive version of this article is the electronic one which can be found at: doi:10.3762/bjoc.9.299 